\title{
Applications of Compositional Analysis with EDS on Battery Materials
}

Kim Larsen ${ }^{1}$, Sam Marks ${ }^{2}$, Matt Hiscock ${ }^{3}$ and Warren Moberlychan ${ }^{4}$

${ }^{1}$ Oxford Instrument NanoAnalysis, High Wycombe, England, United Kingdom, ${ }^{2}$ Oxford Instruments NanoAnalysis, High Wycombe, England, United Kingdom, ${ }^{3}$ Oxford Instruments NanoAnalysis, United States, ${ }^{4}$ Oxford Instruments America, Inc., Massachusetts, United States

Li-ion batteries have been a key enabling technology over the last decade and are considered integral to future green energy developments. With the continued requirements of reduced $\mathrm{CO} 2$ emissions and energy usage, batteries are being developed to support consumer electronics as well as for Electric Vehicles (EVs) and energy storage applications. EVs in particular, are a major point of focus and are driving the next generation of battery technology - with a key challenge being increased energy density - which would eliminate many of the perceived limitations of EVs.

Understanding the composition of the Li-ion battery pre-cursor materials and how the manufacturing processes affect chemistry locally is important for controlling the properties of the final product [1]. Chemical characterisation in the Scanning Electron Microscope (SEM) using Energy Dispersive X-ray Spectroscopy (EDS) is a routine technique in materials science, which is now becoming regularly used for analysis of battery materials and powders used to produce cathode and anode materials [2].

Here we present application examples to demonstrate how EDS can be used to determine the variations in chemistry between particles used for making cathode material and within cathode foils.

Battery pre-cursor powder materials can be analysed using an application optimised automated particle analysis approach (AZtecBattery) to obtain information about contaminants and chemical variations between particles. Particles are automatically identified based on their grey levels in backscatter (BSE) images and X-ray data is automatically acquired from each particle. This combined acquisition of particle morphology and composition means that groups of particles and contaminants can be identified and classified. The approach provides a fast and easy solution for unattended analysis of raw powder materials and precursor materials used in the battery industry for making cathodes and anodes.

We also compare results obtained with a conventional windowed EDS detector (Oxford Instruments Ultim Max) to those from a windowless detector (Oxford Instruments Ultim Extreme), to demonstrate the improvements in spatial resolution which can be achieved when using this technology and analysing at low acceleration voltages: Thereby providing a solution for measuring chemical variations and mapping the light element distribution within NCM cathode material.

An example of this is shown in Figure 1 which shows the comparison between an analysis of a cathode performed at $20 \mathrm{kV}$ with a conventional EDS detector (Ultim Max 170) alongside data collected with a windowless detector (Ultim Extreme) at $5 \mathrm{kV}$. Probe current and analysis parameters were kept constant and the figures clearly demonstrate how the Ultim Extreme provides better spatial resolution data in the same amount of time. 

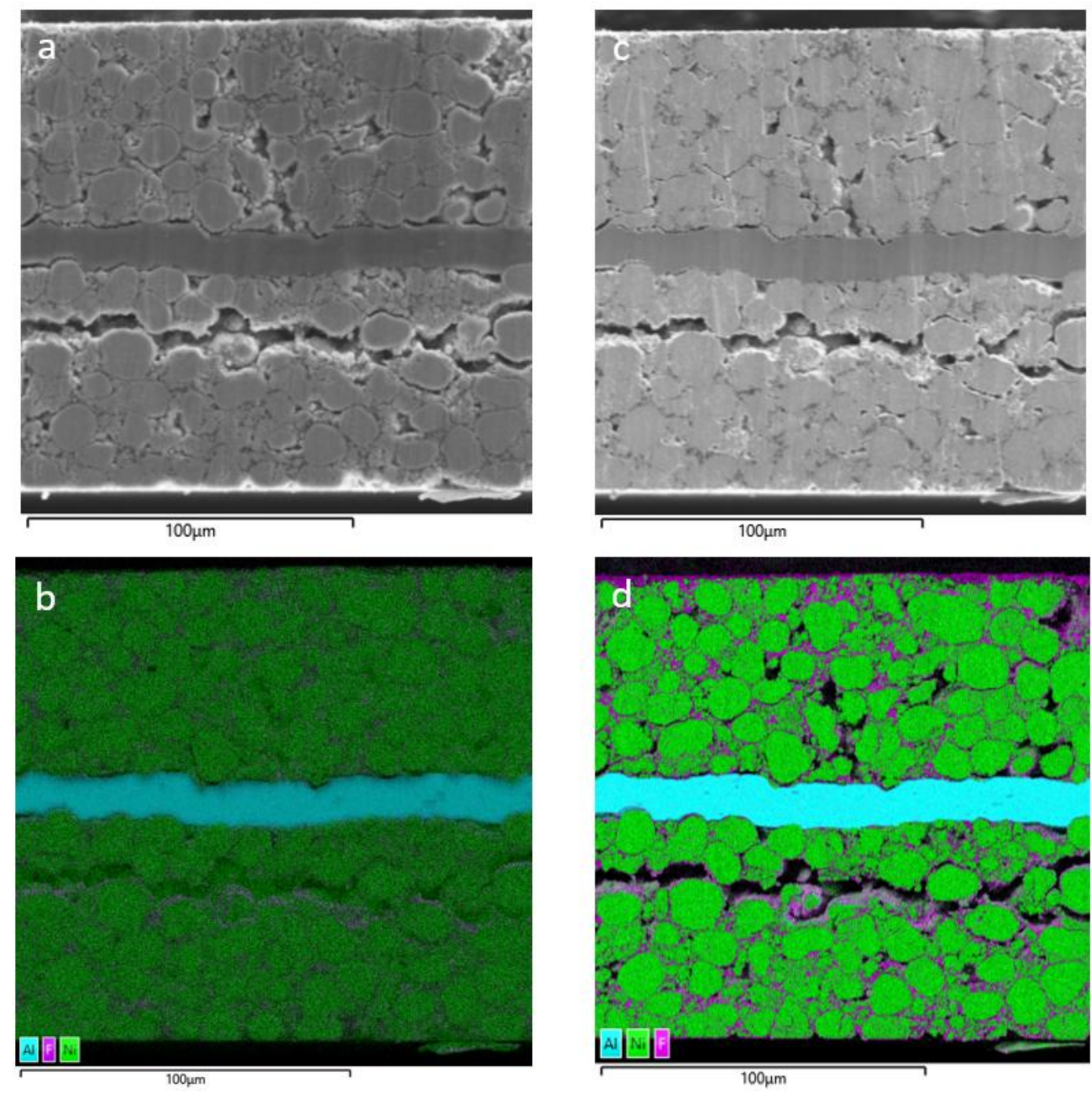

Figure 1. Electron image and EDS Layer maps of an ion milled NCM cathode foil cross section using an Ultim Max 170 EDS detector at 20kV, 300pA (a\&b) and an Ultim Extreme at 5kV, 300pA (c\&d).

\section{References}

[1] Manthiram, A. A reflection on lithium-ion battery cathode chemistry. Nat Commun 11, 1550 (2020). https://doi.org/10.1038/s41467-020-15355-0

[2] Liu et al, Review of recent developments of Insitu/Operando Characterisation techniques for Lithium Battery Research, Advanced Materials 2019 